\title{
A Three-Dimensional Visualization On-line Monitoring System for Large Buildings with OSG and ProE
}

\author{
Yufei $\mathrm{Gu}^{1} \mathrm{a}^{*}$, Xiaomin $\mathrm{Xu}^{2, \mathrm{~b}}$, Hongliang Jia ${ }^{1}$, Honglun Wang ${ }^{1}$, Shaojiang Chen ${ }^{1}$, \\ Yu Lu'
}

${ }^{1}$ Key Laboratory of Space Launching Site Reliability Technology, HaiNan, HaiKou, China

${ }^{2}$ Luoyang Petrochemical Engineering Corporation Ltd/SINOPEC, HeNan, LuoYang, China

ayfgu123@gmail.com, bxuxm.lpec@sinopec.com

Keywords: structural health monitoring, Three-Dimensional Visualization, OSG, ProE.

Abstract. To monitor and show the large buildings' strain, vibration, pressure and other states, a three-dimensional visualization on-line monitoring system based on ProE and the Open Scene Graph (OSG) is proposed in this paper. Firstly, design the buildings' structure using the 3-D modeling tools ProE and then get the digital buildings. Then, with the OSG 3D graphics toolkit, accomplish the zooming, roaming and location functions. Finally, based on the mode of data driven, real-time drawing and rendering the building's all kinds of sensors, and complete the safety condition monitoring for the key positions.

\section{Introduction}

Under the influence of typhoon, earthquake, temperature changes, humidity changes, load changes and other factors, many large buildings will produce vibration, deformation, displacement or collapse, just like cross-sea bridge, dam and other giant buildings. The safety condition monitoring for the buildings' structure is through certain sensor and monitor the key position's state continuous and real-time, and can provide a basis for evaluating their stability and reliability. In addition, the safety monitoring is very important for finding the potential security timely, safe operation, damage monitoring, residual life evaluation for the building. At present, the safety condition monitoring has become an indispensable in the large building construction and maintenance, and become the research hotspot for civil engineering and structural engineering fields.

With the rapid development of software and hardware, virtual reality technology is playing an increasingly important rule in the digital city[1]. Applying this technology into the safety monitoring system, users can get the monitoring result directly and detailed, and can make the maintain and rebuild of building more purposeful. And it can reduce the manpower and material resources greatly due to the maintaining the building. Among this technology, the Open Scene Graph (OSG) has a set of GUI tool kits based on the OpenGL technology and C++ development platform that can develop 3D applications rapidly. At present, with excellent modularity and usability, OSG has been widely applied into producing games, military simulation, scientific visualization and other fields[2-4]. In 2013, Li Si development a 3D simulation system of dam based on OSG platform[5]; In 2014, Yu Yanchao researched the organization and scheduling system for feature model of urban three-dimensional based on OSGEarth[6]; In 2014, Shen Hao designed the 3D visualization system for reservoir based on OSG, with good results[7].

This paper applies the vibration, fiber grating strain, pressure, temperature and wind speed sensors to monitoring the key positions in real time for a certain building. And then design the 3D visualization on-line monitoring system based on the ProE and OSG. The system uses the 3-D modeling tools ProE[8] to design the buildings' structure. Then accomplishes the scenario-driven, 3D displaying and adding sensor models with OSG's graphical capabilities. Finally, with the real data form the all kinds of sensors, rendering the sensor models based on the mode of data driven. 


\section{System design schema}

The on-line monitoring system mainly composed of data acquisition unit, data processing unit, platform driving unit and user interaction unit, the details are presented in Fig.1. The data acquisition unit utilizes all kinds of state sensors in key position of building to collect data in real time, and then sends data to the front end IPC. The IPC transmits data to the after end data processing unit of 3D visualization system. The data processing unit not only collects and loads the building 3D model, but also take charge of processing data for sensor models. It provides the data basis for the system. The platform driving unit just renders and real-time updates the models based on the OSG engine and sensors data, and provide the user with a visual and intuitionistics 3D visualization scene. And based on the user requirements, the interaction unit realizes the function of 3D model translating, roaming, zooming, positioning and warning in the 3D scene.

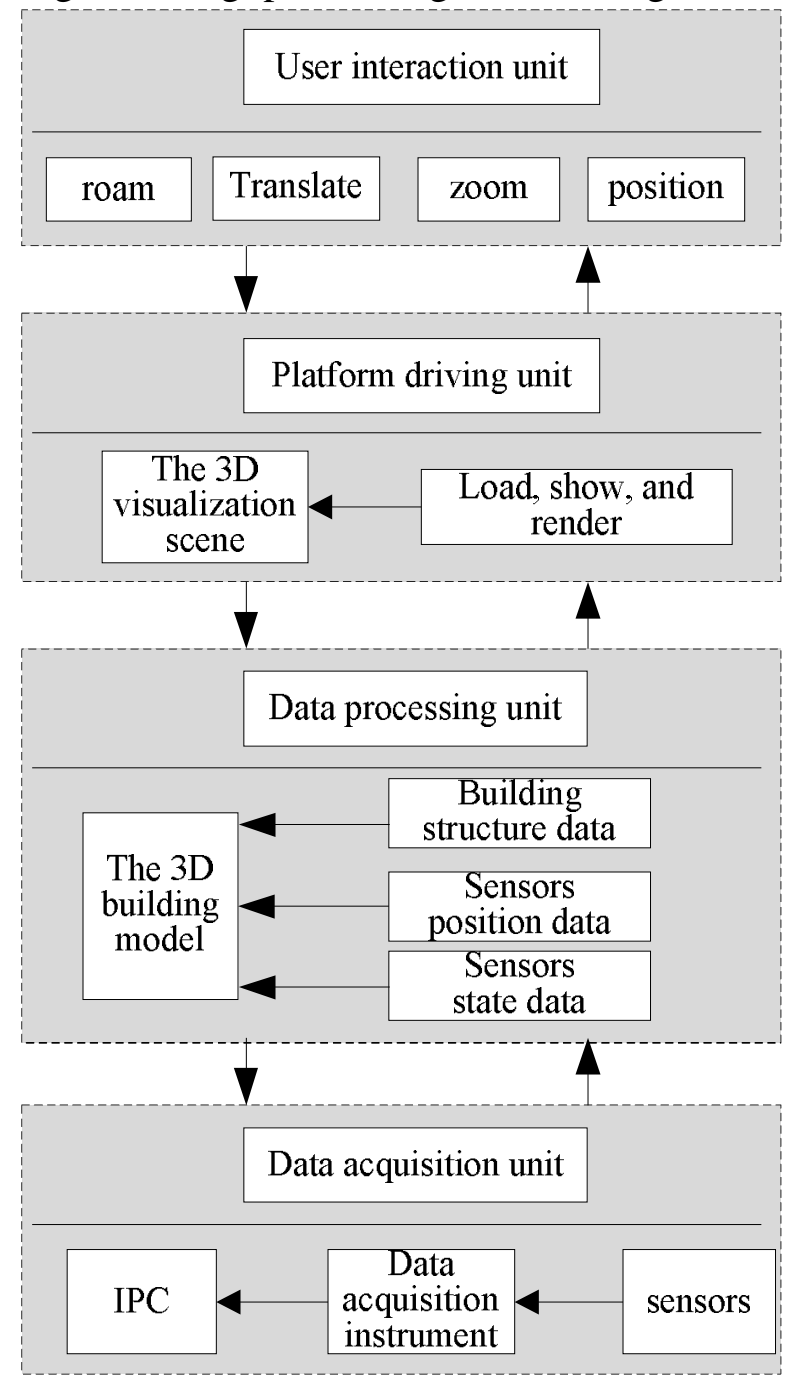

Fig.1 system constitution

\section{D Model design}

The 3D model mainly composed of building model and sensors models in visualization monitoring system.

The building model designing. Large building is typical complicated 3D simulation object, because its structure not just consists of some simple geometries. The workload will be enormous if design the model by purely depend on the OSG code. So, the 3D modeling tools ProE is adopted to 
design the model based on the features of building and its design drawing. In addition, the number of pipe lines for air, gas and power supply is surprising, it will make the model data larger and complicated if drawing the model based on the design drawing absolutely. So this paper focused on the vertical columns, beams, movable fixture, tower cranes and other key positions that with sensors, and obscured the pipe lines that no use for state monitoring.

The sensors models designing. The 3D sensors models are added to the building model with the 3D drawing function of OSG, and based on the installation sites data from the configuration files. And the specific methods are as following.

a. This paper designs different rendering methods for different senors, due to their different features. The pressure sensors adopt different size cylinders to represent different pressure value, as shown in Fig.2(a). And then cover a surface that whose height and color changing constantly according to the different pressure value. The wind speed sensors use the arrow model with different length and direction to represent different wind speed value and wind direction, as shown in Fig.2(b). The vibration sensors utilize the offset and slope of building's stand columns to show the different vibration strength, as shown in Fig.2(c). Finally, the strain sensors adopt the hemispheres with different size and colors to render the different stresses of building, as shown in Fig.2(d).

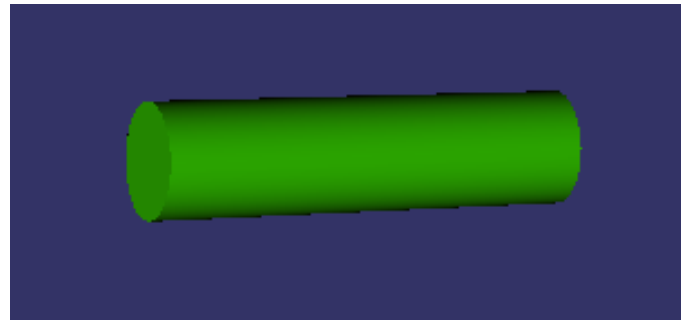

(a) pressure sensors 3D model

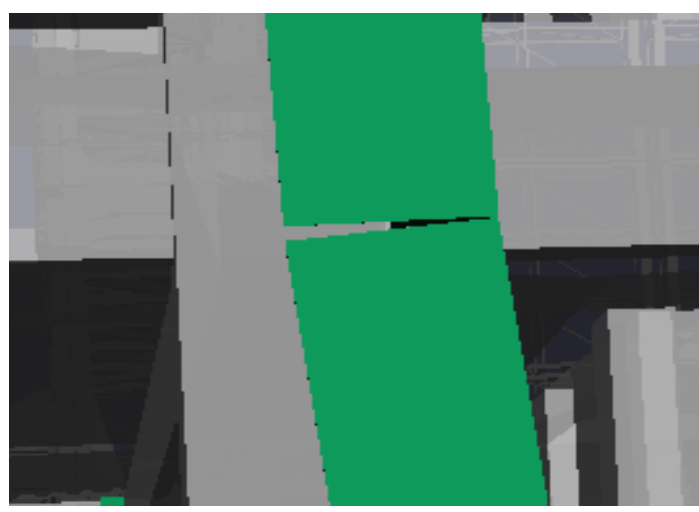

(c) vibration sensors $3 \mathrm{D}$ model

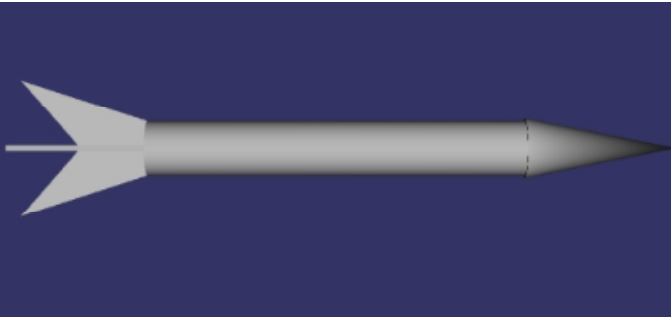

(b) wind speed sensors 3D model

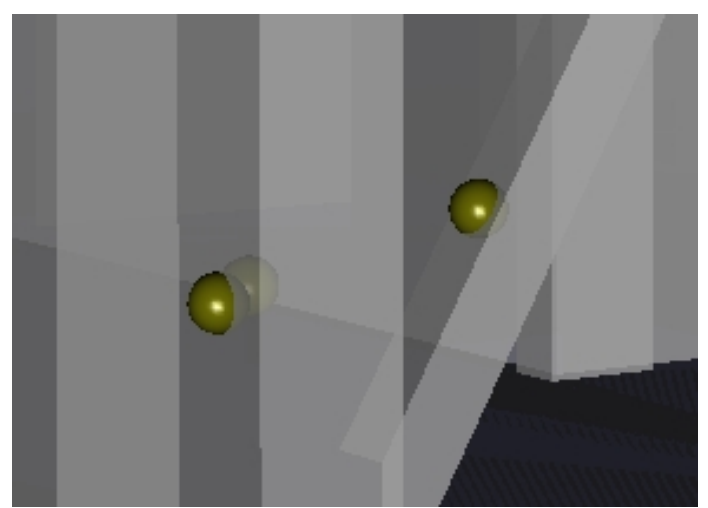

(d) strain sensors 3D model

Fig. 1 The sensor 3D models

b. Firstly, getting the accurate positions that all kinds of sensors in the building from the configuration files. Then, converting these positions to the world coordinate system in OSG based on the OSG coordinate transform connection. Finally, adding these sensor models to the building model based on their corresponding position coordinates.

\section{The optimization schemes and practical application}

Data processing. The visualization system in this paper connects to the front-end IPC directly, and receives the monitoring data real-time. Different sensors have different data encoding formats. So system should decode these data based on their specific encoding format, and then preprocess them. In addition, the system should combine the fiber grating strain sensors data with their corresponding 
temperature based on specific equation[9], and calculate the real strain values. To show these senors state beautiful and normative, this paper normalizes all kinds of sensors data according to real situation.

Locating the key position. To look over the pressure and stress state of building more convenient, this system designed the locating function that can locate the maximum and minimum pressure or stress position. Just clicking the button, above-mentioned positions will be presented inside the window. And users can find where resisting big pressure or having large deformation directly, and avoid translating and zooming continually.

To achieve this function, this paper numbers every sensors firstly, and stores their nodes name and positions into a array. Then compares every kind of sensors data, and gets the maximum and minimum, encodes their ID. Then, system finds and gets above-mentioned nodes name and positions based on their ID. Finally, modulates OSG CameraManipulator's position to show these sensor nodes in window viewport. The modulating process as follows,

1) Firstly, getting the viewport information in current scene, and then computing the distance(Dis_Y) in $\mathrm{Y}$ direction between current viewport and node based on their world coordinates.

2) Then, determining the new viewport coordinate(V) that OSG camera should move to based on Dis_Y and this node's coordinate.

3) Finally, acquiring the OSG scene CameraManipulator, and then setting its' position as (V,C,U) through the setHomePosition() function. Where C stand for node's world coordinate, U stands for the direction that observer locates.

Optimization scheme about render process. To show and operate the 3D building scene smoothly and beautifully, this paper adopts following optimize technology in render process.

1) Converting the .obj format building model ( ProE provided) to the .ive binary file that OSG can load and operate quickly through the osgconv command, and package its corresponding materials and textures.

2) Optimizing the loaded building model with the osgSim::Optimize to reduce the computation complexity.

3) It is a challenge to the system computer performance, because there are hundreds of sensors model must be rendered and updated simultaneously and timely. To improve the fluency of system, this paper references the parallel computation method with computed with Graphics Processing Unit (GPU) to render and update models in parallel with GPU's multicore.

Practical applications. The monitoring result for $\mathrm{X}$ building with large steel structure using this system is shown in Fig. 3, its display worked well and run smoothly and stably. The computing platform is as follows: CPU Intel(3.40GHz), GPU GeForce 605, CPU memory 2GB, GPU memory 512MB, Qt5.4 and win7 64bit. 


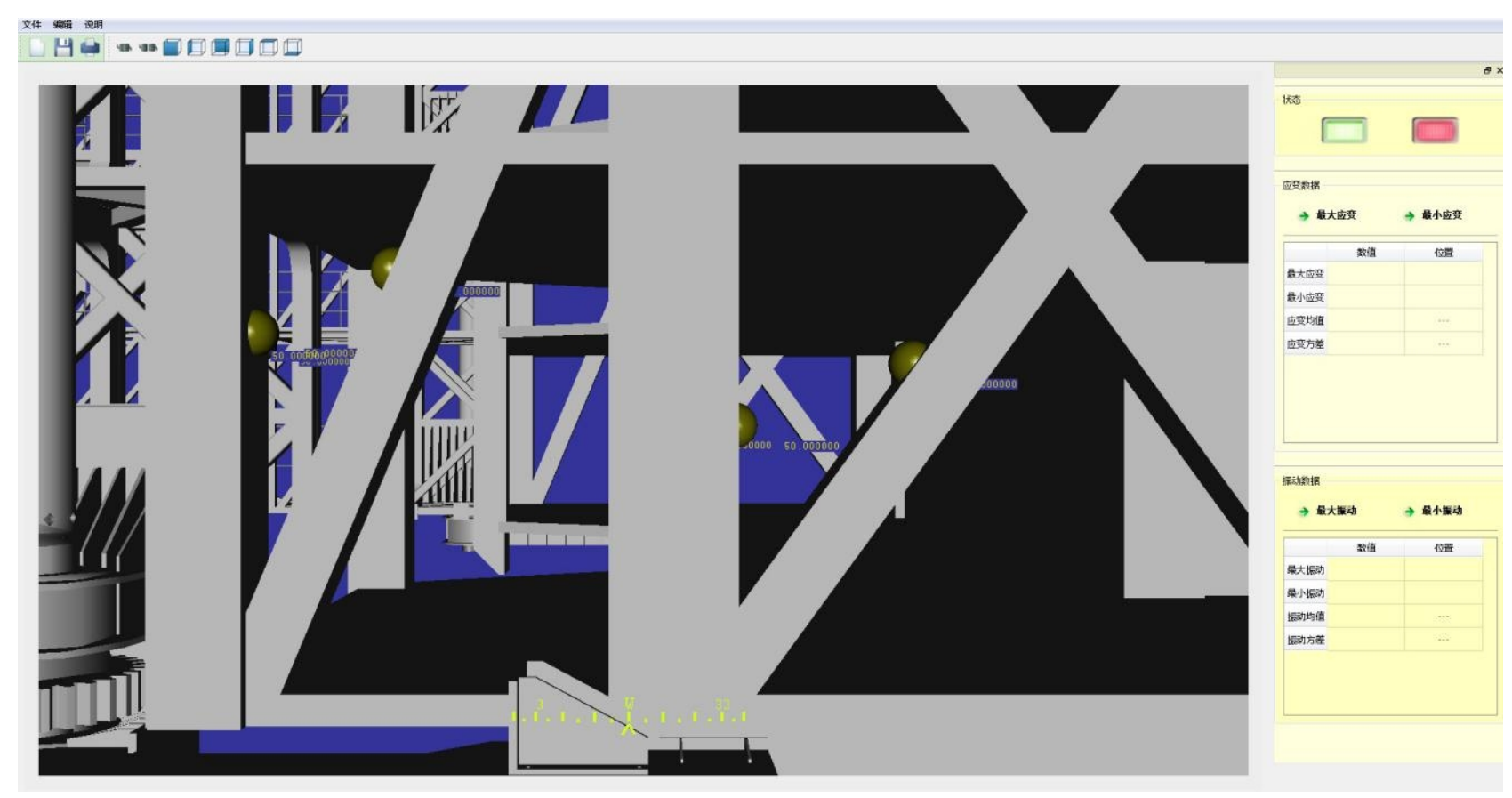

Fig. 3 The monitoring result for $\mathrm{X}$ building

\section{Conclusions}

The structure safe monitoring for large buildings is very important for mastering the health condition and routing maintenance of buildings. And the $3 \mathrm{D}$ visualization on-line monitoring system can show the buildings' safe state intuitively and conveniently, and provide the functions that data analysis and an early warning for dangers. This paper designs and implements the system based on the OSG, ProE and QT development platform, and achieves well monitoring results.

\section{References}

[1] H.B. Dai, X.J. Yue and J.Y. Xu: Applied Mechanics and Materials Vol. 580 (2014), p. 2745.

[2] J. Li, G.J. Li and W. Hou: Lecture Notes in Computer Science Vol. 7719 (2013), p. 282.

[3] Y.F. Yang, W.G. Guo, J. Li and D.F. Chen: Lecture Notes in Computer Science Vol. 7719 (2013), p. 728.

[4] X. K. Meng, Z.X. Hua: E: Computer Simulation Vol. 27 (2010), p. 234. (In Chinese)

[5] S.Li, C.F. Cui and H. Zhang: journal of Water Resources \& Water Engineering Vol. 24 (2013), p. 85. (In Chinese)

[6] Y.C. YU, H.W. XU and X.D. WU: GEOMATICS \& SPATIAL INFORMATJON TECHNOLOGY Vol. 37 (2014), p. 63.(In Chinese)

[7] H. Shen, Q.K. Liu and W.Q: Image Processing and Multimedia Technology Vol. 33 (2014), p. 32. (In Chinese)

[8] J.W. Guo, J.Q. Fan and Y. Chen: Advanced Materials Research Vol.655-657 (2013), p. 1029.

[9] Zh.Y. Zhao, Sh. Zhang and Y.S. Yu: Microwave and Optical Technology Letters Vol.43(2004), p.324. 